\title{
The role of B cell Activating Factor (BAFF) expression on pathogenesis of nasal polyp in chronic rhinosinusitis with nasal polyposis*
}

\author{
Young Hoon Yoon', Jun Jin', Ki Ryun Kwon', Sung Ha Kim', Ki-Sang Rha',2, \\ Yong Min Kim ${ }^{1,2}$ \\ Rhinology 52: 390-396, 2014 \\ DOl:10.4193/Rhino13.154 \\ ' Department of Otorhinolaryngology-Head and Neck Surgery, Chungnam National University, School of Medicine, Daejeon, South *Received for publication: \\ Korea \\ October 3, 2013 \\ ${ }^{2}$ Research Institute for Medical Sciences, Chungnam National University, School of Medicine, Daejeon, South Korea \\ Accepted: February 19, 2014
}

\begin{abstract}
Aim: B cells, plasma cells, and local immunoglobulins, are important as a mucosal immune barrier function, and tend to increase in patients with chronic rhinosinusitis with nasal polyposis (CRSwNP). However, their association with eosinophils' aggregation has not been fully understood. The objectives of this study was to explore whether BAFF expression in the subepithelial area in nasal polyp tissues of CRSwNP was associated with eosinophils' accumulation, and also to evaluate cells which cells produce BAFF.

Methodology: Immunohistochemical and immunofluorescence staining were used to analyse expression of BAFF, CD20, immunoglobulin A (IgA) and a proliferation inducing ligand (APRIL) on nasal tissues from CRSwNP patients and control subjects. To identify the relationship between BAFF and tissue eosinophilia, CRSWNP subjects were divided into eosinophilic polyp and non-eosinophilic groups. Double immunofluorescence analysis for BAFF and CD11c or CD11b was performed to identify cells producing BAFF.
\end{abstract}

Results: The numbers of BAFF, CD20, and IgA-positive cells in the subepithelial area were significantly higher in the CRSwNP group (both eosinophilic polyps and non-eosinophilic polyps) compared to the control group, and also significantly higher in eosinophilic polyps compared to non-eosinophilic polyps. There were statistically significant correlations between the number of BAFF and CD20-positive cells, CD20 and IgA-positive cells, and BAFF and IgA-positive cells. CD11 b-positive cells were co-localized with BAFF positive cells.

Conclusion: The subepithelial expression of BAFF was associated with increased number of B cells and plasma cells, and increased production of IgA in the patients with CRSwNP, especially eosinophilic nasal polyps. Therefore, BAFF-induced IgA production may be associated with eosinophils' aggregation and degranulation, which cause aggravation of tissue inflammation and finally polyp formation. The expression of BAFF in the subepithelial area may be associated with innate inflammatory cells (CD11 b+ cells), such as monocytes, granulocytes, macrophages, and natural killer cells.

Key words: B-Cell Activating Factor, B-lymphocytes, Immunoglobulin A, sinusitis, nasal polyps

\section{Introduction}

Chronic rhinosinusitis with nasal polyposis (CRSwNP) is a chronic inflammatory disease of nasal and paranasal sinus mucosa and its precise etiologies are still unclear ${ }^{(1)}$. It has been known that patients with nasal polyps are more likely to be associated with Th2-based inflammation, an increase of tissue eosinophils, and polyclonal immunoglobulin-E (lgE) production ${ }^{(2,3)}$. Recent studies have reported increased infiltration of B cells and plasma cells, and production of local Ig, especially antigenspecific $\lg \mathrm{E}$, IgG and $\lg \mathrm{A}$ in the nasal polyps of patients with CRSwNP ${ }^{(4-6)}$. Although recent evidence showed that $B$ cell and Ig in the tissue play an important role in pathogenesis of CRSWNP, 
their exact mechanisms have not been fully understood.

B cell-activating factor of the TNF family (BAFF) and a proliferation inducing ligand (APRIL) have recently been identified as members of the TNF superfamily ${ }^{(7)}$. They are known to play important roles in B cell survival, proliferation, and maturation ${ }^{(8)}$. BAFF is an important regulator of immunoglobulin class-switch recombination and production in $B$ lymphocytes by $T$ cell-independent or-dependent manner ${ }^{(9,10)}$. Overproduction of BAFF in nasal polyps may contribute to the pathogenesis of CRSwNP through the local infiltration of $B$ cells and IgA production ${ }^{(5)}$. $\lg A$ in the mucosa acts as the defensive factor against infectious microorganisms, and is also known as an important immune factor for maintaining and controlling the homeostasis of symbiotic bacteria ${ }^{(11)}$. It is known that locally elevated antigen-specific $\lg \mathrm{E}$ and plasma cells increases the degranulation of eosinophils and aggravate local mucosal inflammation in nasal polyps ${ }^{(3,6)}$. However, it remains unclear whether locally elevated IgA actually increases the degranulation of eosinophils and basophils in the nasal polyps. In the blood circulation, IgA has been known to interact with an Fc receptor called FcaRI (CD89): expressed on the surface of the immune effector cells to initiate inflammatory reactions ${ }^{(12)}$. Ligation of FcaRl by IgA containing immune complexes causes antibody-dependent cell-mediated cytotoxicity (ADCC): degranulation of eosinophils and basophils, phagocytosis by monocytes, macrophages, and neutrophils ${ }^{(12)}$.

Although there have been many studies relating BAFF and APRIL with various types of auto-immune diseases, B-cells related malignancy, and obesity, few studies have explored their role in nasal polyps of CRSwNP patients ${ }^{(13-15)}$. In the present study, we explored possible differences in the expression of BAFF and APRIL between eosinophilic polyps and non-eosinophilic polyps, and whether the expressions of these factors were associated with eosinophils aggregation. In addition, we also examined potential cells involved in the production of BAFF in the subepithelial area.

\section{Materials and methods}

\section{Patients}

A total of 45 patients were enrolled in this study, of which 30 had CRSwNP, while 15 were undergoing septoplasty for nasal septal deformity. The nasal polyps of CRSwNP were subclassified into eosinophilic polyps (EP) and non-eosinophilic polyps (NEP) according to the result of hematoxylin and eosin (H\&E) stain ${ }^{(4)}$. The polyp was defined as an EP if the proportion of eosinophils exceeded $10 \%$ of the total infiltrating cells in the tissue, if lower, it was defined as a NEP. Inferior turbinate mucosa from control subjects and nasal polyps from CRSwNP patients were collected for immunohistochemical or immunofluorescent staining. The diagnosis of sinus disease was based on history
Table 1. Patient characteristics.

\begin{tabular}{lccc|}
\hline & Controls & $\begin{array}{c}\text { Non- } \\
\text { eosinophilic } \\
\text { CRSwNP }\end{array}$ & $\begin{array}{c}\text { Eosinophilic } \\
\text { CRSwNP }\end{array}$ \\
\hline $\begin{array}{l}\text { Total of subjects, } \mathrm{n} \\
\text { Sex, male, } \mathrm{n}\end{array}$ & 15 & 15 & 15 \\
\hline $\begin{array}{l}\text { Age, yr mean } \\
\text { (range) }\end{array}$ & 12 & 9 & 11 \\
\hline $\begin{array}{l}\text { Patients with Atopy, } \\
\mathrm{n}\end{array}$ & 6 & $40.27(13-74)$ & $41.60(15-60)$ \\
\hline $\begin{array}{l}\text { Lund-Mackay CT } \\
\text { score }\end{array}$ & - & 5 & 10 \\
\hline $\begin{array}{l}\text { Infiltration of tissue } \\
\text { eosinophils } \%\end{array}$ & - & $16.3 \pm 4.5$ & $15.4 \pm 3.5$ \\
\hline
\end{tabular}

CRSwNP, chronic rhinosinusitis with nasal polyps.

clinical examination, nasal endoscopy, and computed tomography of the paranasal sinuses. Reasons for exclusion included use of oral or nasal corticosteroids, use of other medications (e.g., antibiotics or antileukotrienes) for 4 weeks prior to sample collection, histories of recent upper respiratory tract infection, and revision surgeries.

Details of the subjects' characteristics are included in Table 1. A written informed consent was obtained from each study participant before enrollment into the study. The study was approved by the Institutional Review Board of the Chungnam National University Hospital.

Immunohistochemistry (IHC) and immunofluorescence (IF) study

Paraffin-embedded tissue samples were soaked in xylene to remove the paraffin wax and then sequentially in solutions of $100 \%, 95 \%$, and $70 \%$ ethanol for rehydration. Antigen unmasking was performed by heating the slides in a Decloaking Chamber (Biocare Medical, Concord, CA, USA) to $120^{\circ} \mathrm{C} . \mathrm{H}_{2} \mathrm{O}_{2}$ block (Lab Vision, Fremont, CA, USA) and protein block (Dako Cytomation, Carpinteria, CA, USA) were then applied to the tissue to block endogenous peroxidases and prevent non-specific protein binding, respectively. Primary antibodies were applied at the following concentration: 1:200 rabbit anti-BAFF antibody (Abcam, Cambridge, MA, USA), and 1:200 rabbit anti-APRIL antibody (Prosci inc., Poway, CA, USA). Primary antibodies were applied for $24 \mathrm{hrs}$ at $4^{\circ} \mathrm{C}$ and the slides were rinsed thrice with PBS before incubating for $2 \mathrm{hrs}$ with appropriate secondary antibodies. After further washing, the slides were stained with DAB (Vector, Burlingame, CA, USA), and counterstained with hematoxylin. 

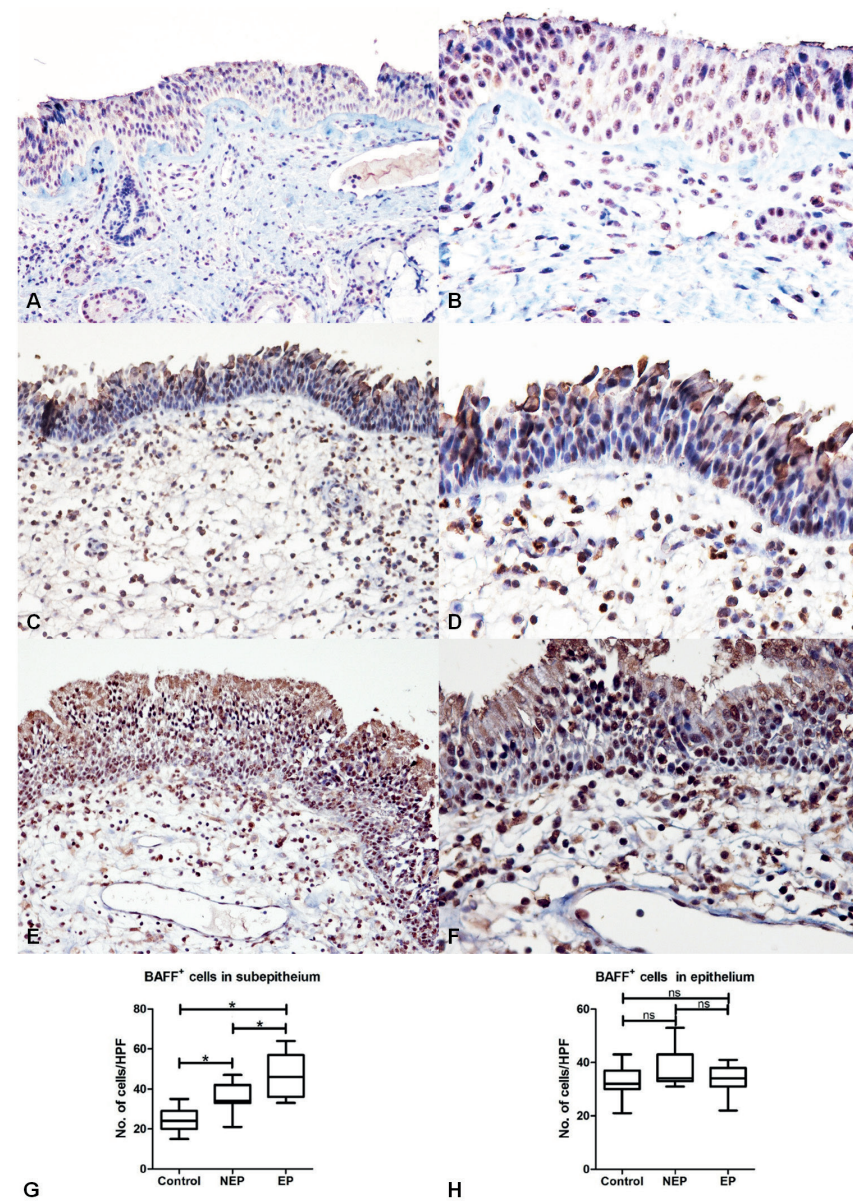

Figure 1. Immunohistochemical stain for $B$ cell activating factor (BAFF) ( $A, C, E: 200 x / B, D, F: 400 x$ ). A and B are control. C and D are non-eosinophilic polyp (NEP). E and $F$ are eosinophilic polyp (EP). Differences of the number of $B$ cell activating factor positive cells among the group in subepithelium (G). Differences of the number of $B$ cell activating factor positive cells among the group in epithelium $(H) .{ }^{*}: P<0.05$, ns: statistically non-specific).

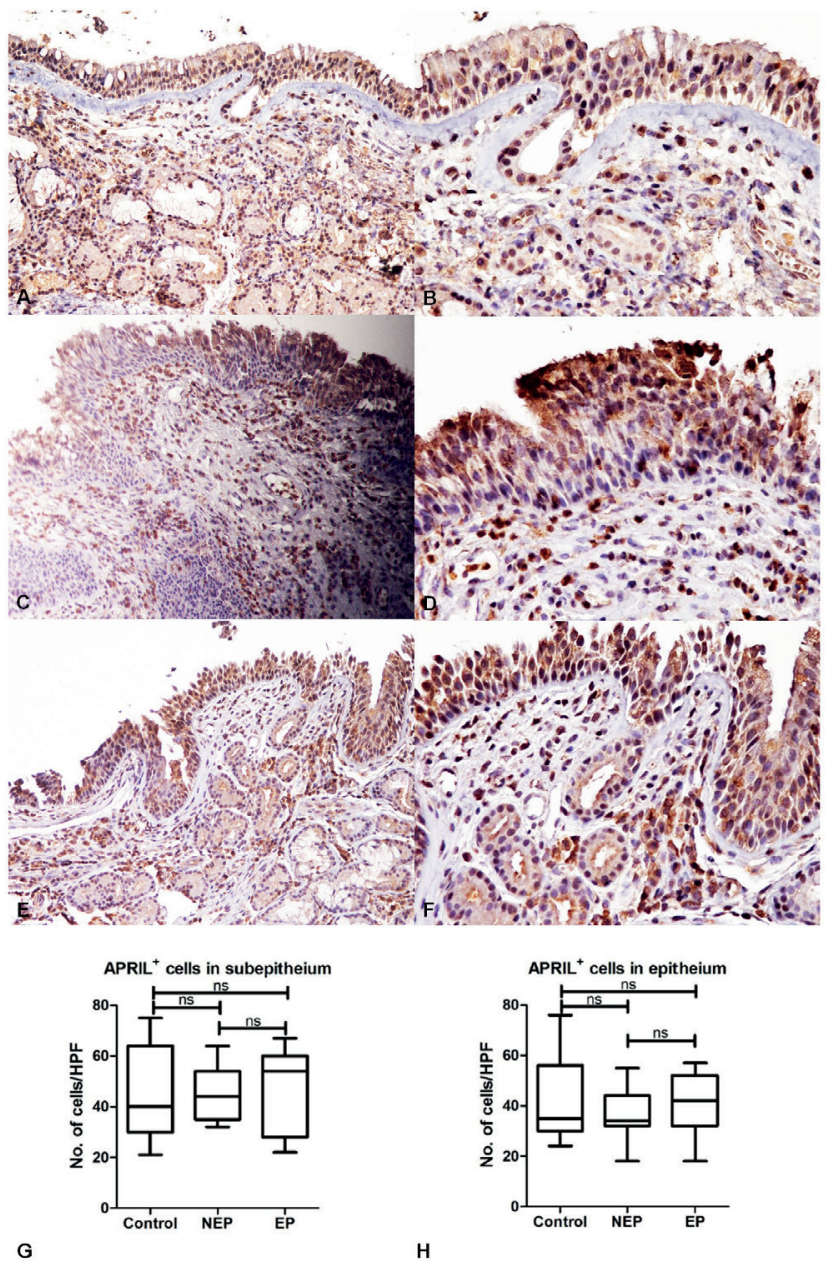

Figure 2. Immunohistochemical stain for a proliferation inducing ligand (APRIL) (A, C, E: 200x/ B,D,F: 400x). A and B are control. $C$ and $D$ are noneosinophilic polyp (NEP). E and $F$ are eosinophilic polyp (EP). Differences of the number of a proliferation inducing ligand positive cells among the group in subepithelium (G). Differences of the number of a proliferation inducing ligand positive cells among the group in epithelium $(\mathrm{H})$. (ns: statistically non-specific).
Immunofluorescence (IF) study was performed using rabbit polyclonal antibody/FITC (DakoCytomation, Glostrup, Denmark) for the human-IgA and mouse monoclonal antibody (BD Biosciences) for the human-CD20. Cy2-streptoavidin (green) was added for $\mathrm{CD} 20$ as secondary reagent.

Double immunofluorescence experiments were performed using Cy3-biotin Rb (red)-conjugated anti-rat IgG for BAFF (Abcam) or Cy3-biotin Rb (red)-conjugated anti-rabbit IgG for BAFF-R (Prosci inc.) and Cy2-Streptoavidin (green)-conjugated anti-mouse lgG for CD20 (BD Biosciences). The other experiments were performed using Cy3-biotin $\mathrm{Rb}$ (red)-conjugated anti-rat IgG for BAFF (Abcam) and Cy2-Streptoavidin (green)conjugated anti-mouse Ig for CD11b (AbD serotec, Kidlington, UK) or Cy2-Streptoavidin (green)-conjugated anti-mouse IgG for CD11c (Santa Cruz Biotechnology Inc, Dallas, TX, USA). After rinsing with PBS, the 4'-, 6'-diamidino-2 phenlylindole dihydrochloride (DAPI, Santa Cruz biotechnology) was used for nucleus staining. Species- and subtype-matched antibodies were used as negative controls. The slides were subsequently observed under a fluorescence microscope (Olympus, Tokyo, Japan).

The numbers of BAFF-positive, APRIL-positive, CD20-positive, and IgA-positive cells were counted per high-powered field $(\mathrm{HPF}, \times 400)$ at three different sites. The numbers of BAFF-positive and APRIL-positive cells were also counted in both epithelial layer and sub-epithelial layer. The BAFF+CD20+, BAFF-R+CD20+, $B A F F+C D 11 b+$, and $B A F F+C D 11 c+$ cells were observed in the eosinophilic polyps to localize BAFF or BAFF-R on different type of cells. The slides were examined by three pathologists who were blinded for the patients' clinical information. 

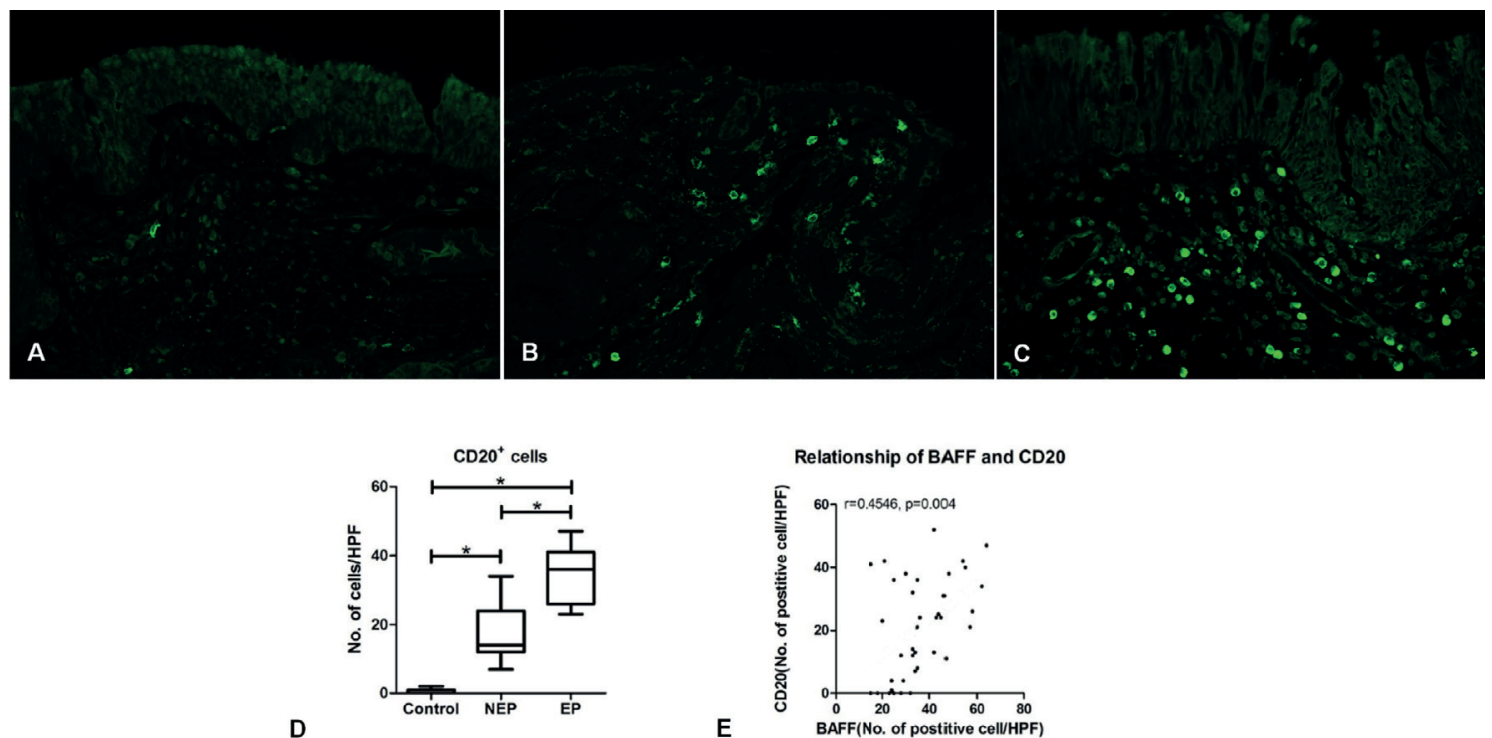

Figure 3. Immunofluorescence assay (x400) for CD20 on control (A), noneosinophilic polyp (NEP) (B), and eosinophilic polyp(EP) (C). Differences of the number of $C D 20$ positive cells among the group (D). Correlation between $B$ cell Activating Factor and CD20 (E). $\left.{ }^{*}: P<0.05\right)$

\section{Statistical analysis}

All statistical analyses were performed using SPSS (version 18.0, SPSS, Inc., Chicago, IL, USA). One-way ANOVA with post-mortem analysis using Tukey method, and Spearman correlation tests were applied with significance levels set at $p<0.05$.

\section{Results}

Expression patterns of BAFF and APRIL in nasal mucosa Expressions of both BAFF and APRIL were localized in the tissue using immunohistochemistry. BAFF-positive cells were detected in both epithelial layers and subepithelial area from three groups (Figure 1A-F). The estimates of the BAFF+ cells in the epithelial layer were $31.47 \pm 9.00$ in the control, $38.07 \pm 6.35$ in the NEP, and $35.27 \pm 8.49$ in the EP group, while the BAFF+ cells in subepithelial area were $24.27 \pm 5.90$ in the control, $37.00 \pm 12.77$ in the NEP, and $47.47 \pm 10.36$ in the EP group. There were no significant differences in BAFF-positive cells within the epithelial layers (Figure $1 \mathrm{H}$ ). However, in the subepithelial area, the BAFF+ cells were significantly higher in either EP or NEP compared to control group (Figure 1G) $(p<0.001)$. In addition, BAFF+ cells were significantly higher in EP compared to NEP (Figure 1G) ( $p$ $<0.05)$.

The APRIL-positive cells were also detected in epithelial layers, subepithelial area and submucosal gland (Figure 2A-F). The numbers of the APRIL+ cells in the epithelial layer were $43.00 \pm$ 17.32 in the control, $36.87 \pm 11.39$ in the NEP, and $39.87 \pm 10.94$ in the EP group, while the numbers in the subepithelial area were $45.07 \pm 18.10$ in the control, $46.07 \pm 9.72$ in the NEP, and $46.13 \pm 16.41$ in the EP group. There were no significant differen-

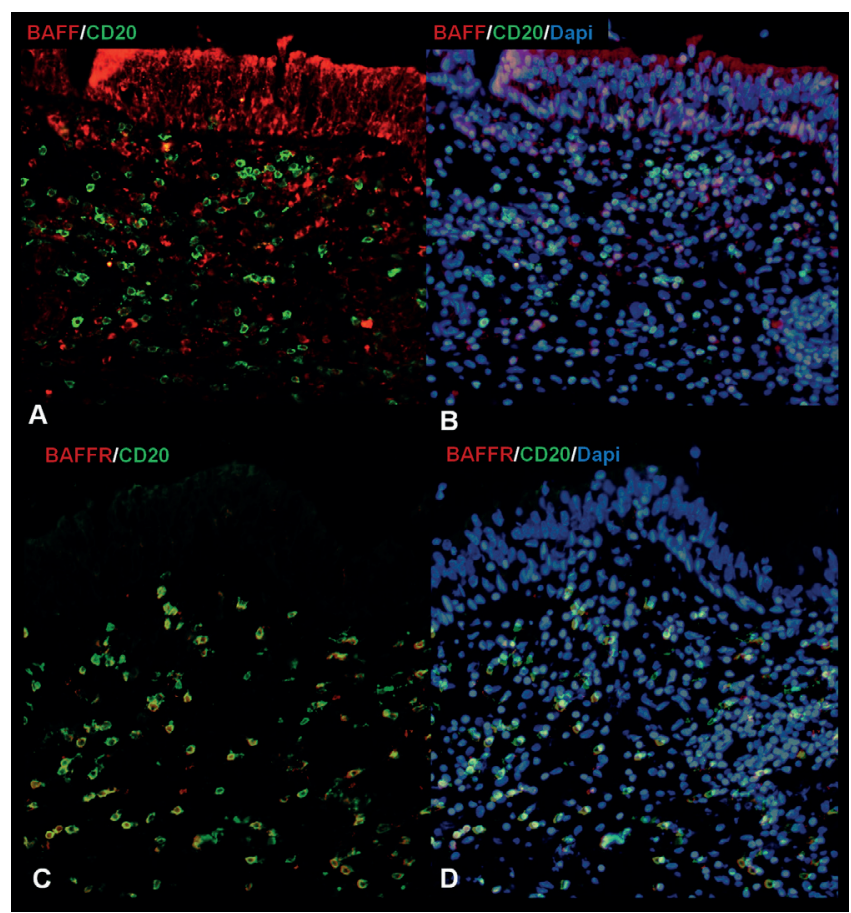

Figure 4. Representitive images of double immunofluorescence assay in eosinphilic polyps; B cell activating factor (BAFF) with CD20 (x400) (A), $B$ cell activating factor with CD20 and Dapi (x400) (B), B cell activating factor receptor (BAFFR) with CD20 (x400) (C) and B cell activating factor receptor with CD20 and Dapi (x400) (D).

ces among the groups within either the subepithelial (Figure 2G) or epithelial layers (Figure $2 \mathrm{H}$ ). 

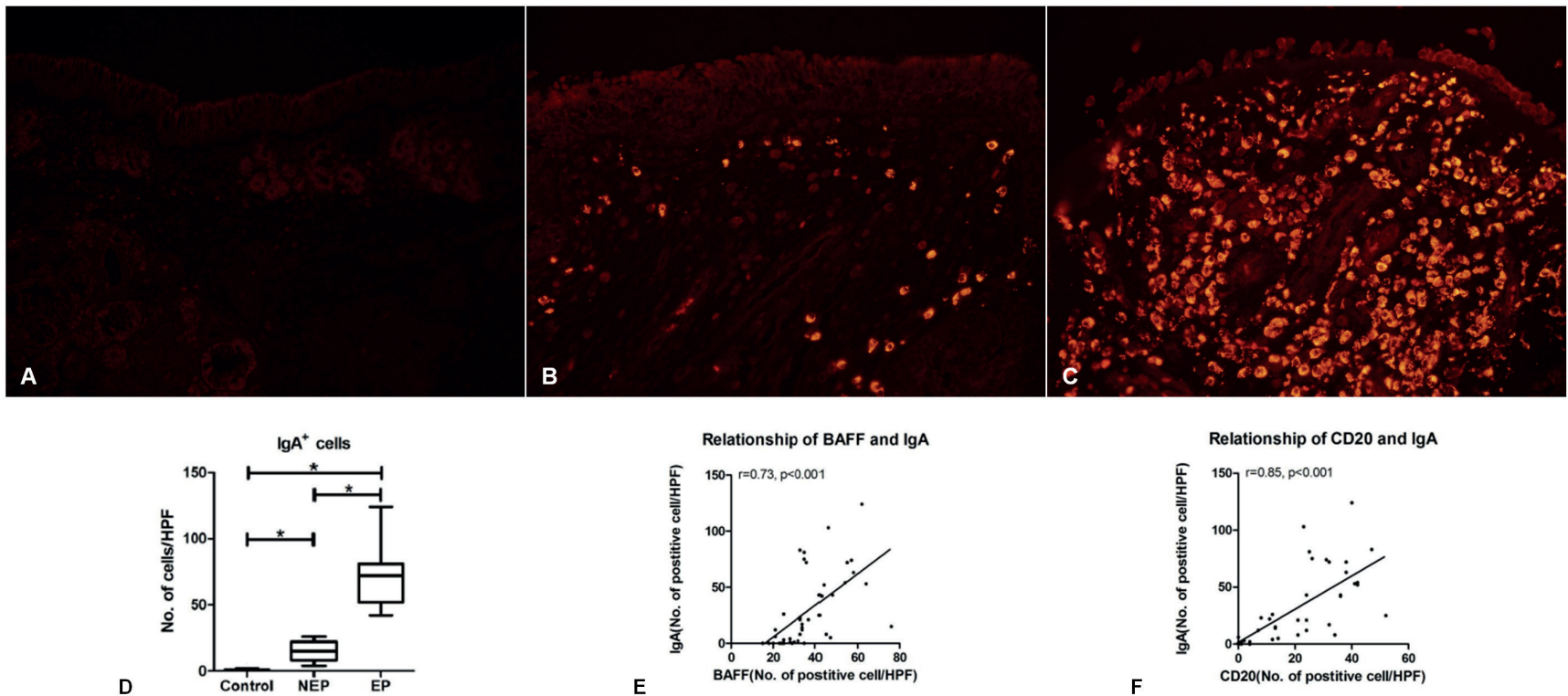

Figure 5. Immunofluorescence assay (x200) for IgA on control (A), non-eosinophilic polyp (NEP) (B), and eosinophilic polyp (EP) (C). Differences of the number of $\lg A$ positive cells among the group (D). Correlation between $B$ cell activating factor (BAFF) and IgA (E). Correlation between CD20 and IgA (F). $\left({ }^{*}: \mathrm{P}<0.05\right)$

\section{Distribution of CD20 positive cells in the tissue}

Since the expression of BAFF was significantly higher in nasal polyps, an IF stain was conducted to evaluate if CD20 positive cells ( $B$ cells) were also higher in nasal polyps (Figure $3 \mathrm{~A}-\mathrm{C}$ ). The estimates of the CD20+ cells were $(0.8 \pm 1.42)$ in the control, $19.87 \pm 12.11$ in the NEP, and $34.73 \pm 7.53$ in the EP group. The CD20+ cells were significantly higher in both EP and NEP groups compared to the control group (Figure 3D) $(p<0.001)$. In addition, the numbers of the CD20+ cells were significantly higher in the EP compared to the NEP group (Figure 3D) ( $<<0.001)$. The number of the $\mathrm{CD} 20+$ cells also showed a statistically significant correlation with the number of BAFF positive cells in the subepithelial area (Figure $3 E)(r=0.4546, p<0.005)$. Therefore, the expression of BAFF may be associated with accumulation of $B$ cells in the nasal polyps, especially eosinophilic polyps. Eosinophilic polyps were stained with CD20 and BAFF or BAFF-R in a double IF study. Although the expression of BAFF did not co-localize with CD20+ cells (Figure 4A, B), BAFF-R expression is co-localized with CD20+ cells in the eosinophilic polyps (Figure 4C, D).

\section{Detection of IgA-positive cells}

$\lg \mathrm{A}+$ cells were increased tremendously in nasal polyps, especially EPs compared to control mucosa (Figure 5A-C). The estimates of the $\lg A+$ cells were $0.67 \pm 1.68$ in the control, 15.53 \pm 7.29 in the NEP, and $68.93 \pm 23.13$ in the EP group. IgA+ cells were significantly higher in the NEP and the EP group compared to the control group $(p<0.001)$. Furthermore, IgA+ cells were significantly higher in the EP compared to the NEP group (Figure $5 D)(p<0.001)$. There was significant correlation between the

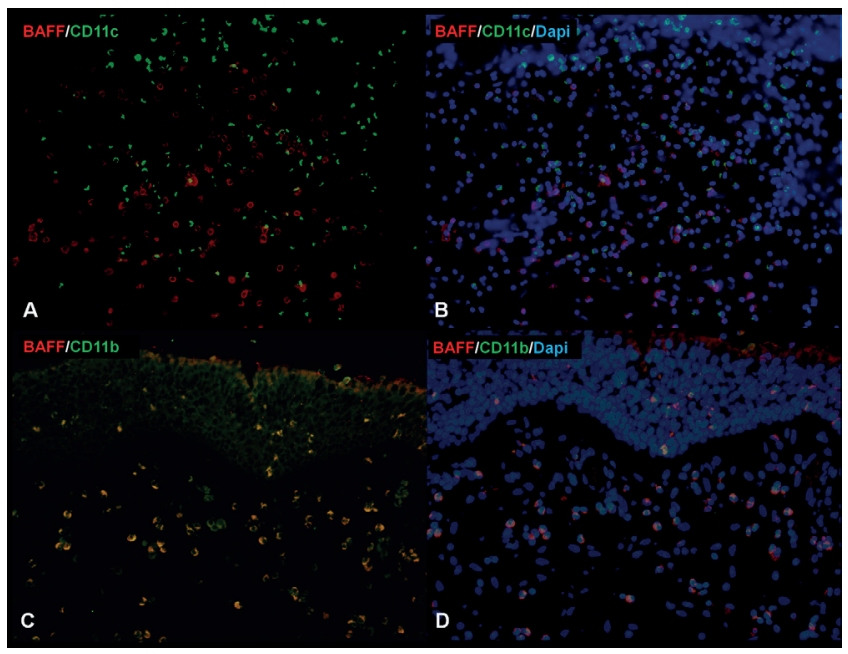

Figure 6. Representitive images of double immunofluorescence assay on eosinophilic polyp; B cell activating factor (BAFF) with CD11C ( $x 200)(A)$ and B cell activating factor with CD11C and Dapi (x200) (B), B cell activating factor (BAFF) with CD11b (x200) (C) and B cell activating factor with CD11b and Dapi (x200) (D).

number of IgA+ cells and the BAFF+ cells (Figure $5 E)(r=0.73$, $p<0.001)$ or CD20+ cells (Figure 5F) $(r=0.85, p<0.001)$.

\section{BAFF is mainly produced by CD11b+cells in the lamina} propria of nasal polyps

It is generally known that BAFF is usually produced by epithelial cells, monocytes, fibroblasts, macrophages, and other submucosal stromal cells ${ }^{(16-18)}$. Since only subepithelial expression of BAFF was significantly higher in the EPs compared to the 
NEPs or the control group in the present study (Figure 1G), we investigated which type of inflammatory cells produces BAFF in the lamina propria. Most of the BAFF positive cells were not Colocalized with CD11C+ cells (Figure 6A, B), but with CD11b+ cells (Figure 6C, D) in both epithelial layers and subepithelial area of EPs using double immunofluorescent staining.

\section{Discussion}

Unlike our study, previous studies evaluated expression of BAFF using different groups. They showed that BAFF-positive cells in the subepithelial area were significantly higher in polyp tissue of a CRSwNP group compared to the uncinate process mucosa of a CRSsNP (CRS without NP) or control group ${ }^{(5)}$. Nevertheless, our study demonstrated that BAFF-positive cells in the subepithelial area were also significantly higher in the CRSwNP group, especially in eosinophilic nasal polyps, compared to the control group. In addition, there were no significant differences in epithelial expression among the groups ${ }^{(5)}$.

It has been known that most Asian patients have neutrophilic, IL-17-biased polyps unlike Caucasian patients with eosinophilic predominant polyps ${ }^{\left({ }^{19}\right)}$. However, eosinophilic infiltrations can be also predominant in some polyps found among Asians. Our study cohort showed that nasal polyps from patients with CRSwNP can be classified as EPs and NEP. Furthermore, our result indicated that the number of BAFF-positive cells in the lamina propria may be associated with eosinophils' aggregation in nasal polyps, because BAFF-positive cells are significantly higher in eosinophilic polyps compared to non-eosinophilic polyps. In the human intestine, epithelial cells release APRIL after sensing bacteria through Toll-like receptors (TLRs) and further increased APRIL production by activating dendritic cells via thymic stromal lymphopoietin (TSLP) ${ }^{(9)}$. Therefore, the intestinal mucosa maintains a high baseline expression of APRIL, and this level of APRIL involves an IgA class switching in B-cells through a T cell independent pathway ${ }^{(9)}$. In our study, nasal epithelial cells and subepithelial stromal cells also preserved a high level of APRIL expression regardless of the group, with no significant differences. This result indicates that the level of APRIL in the nasal mucosa is not associated with nasal polypogenesis. It also demonstrates, that BAFF may be more important than APRIL in triggering the induction of immunoglobulin class-switch recombination and production induced by pathogens in nasal airways ${ }^{(5)}$.

BAFF is known to function by binding with three receptors; the BAFF receptor (BAFF-R), the B-cell maturation antigen (BCMA), and the transmembrane activator and the $C A M L$ interactor $(\mathrm{TACl})^{(18)}$. BAFF binds to BAFF-R with high affinity, and BAFF-R was co-localized on the surface of $B$ cells in our study (Figure $4 C, D)$. APRIL, on the other hand, is known to act through the combination with $\mathrm{TACl}$ and $\mathrm{BCMA}{ }^{(20)}$. These receptors are uniquely expressed on B-cells and plasma cells, and are known to maintain homeostais and control the activity of the B-cell through binding with BAFF or APRIL ${ }^{(18,20)}$. Enhanced expression of BAFF in the subepithelial area of nasal polyps may be due to increased binding of BAFF to its receptor on $B$ cells or plasma cells. However, BAFF did not co-localize with $B$ cells in our study (Figure $4 A, B$ ), and this may indicate that other cells in lamina propria of eosinophilic nasal polyps may produce BAFF.

Our study showed BAFF expression was significantly correlated with B-cell aggregation (Figure $3 \mathrm{E}$ ) and IgA production (Figure $5 \mathrm{E})$. Mucosal immunoglobulin can activate the isotype-specific Fc receptors on the surface of phagocytes or other immune cells and accelerate the defensive capacities against infiltrated pathogens ${ }^{(11)}$. In human, there are three isotype-specific Fc receptors; three types IgG-specific Fc receptors (FcyRI, FcyRII, FcyRIII); an IgA-specific Fc receptor (FcaRI); and a high-affinity lgE Fc receptor (FcERil). These receptors are expressed on various types of immune cells in the lamina propria of the mucosa ${ }^{(21,22)}$. When the pathogenic antigen that is surrounded by immunoglobulin is combined with these Fc receptors, it gets eliminated through phaygocytosis, secretion and degranulation of antimicrobial agents, including the production of activated oxygen species ${ }^{(11,23)}$. In the present study, IgA was detected mainly on the cellular surface in the subepithelial area of eosinophilic nasal polyps (Figure 5C). Our results indicate that the subepithelial accumulation of $\lg \mathrm{A}$ and activation of various immune cells through Fc receptors may be involved in pathogenesis of eosinophilic nasal polyps.

It has been known that subepithelial BAFF is produced by several stromal cells such as monocytes, histiocytes, fibroblasts, epithelial cells of submucosal glands and blood vessels ${ }^{(18,24,25)}$. Our study showed that subepithelial BAFF was produced mainly by the CD11b-positive cells, and not by CD11c cells in eosinophilic nasal polyps. This implies that CD11b-positive cells play a pivotal role in producing BAFF, controlling the inflammatory responses, and in the pathophysiology of nasal polyps. CD11b is known as integrin alpha $\mathrm{M}$ which is one protein subunit that forms the heterodimeric integrin alpha M beta-2 (aM 32 ) molecule ${ }^{(26)}$. aM 32 is expressed on the surface of many leukocytes involved in the innate immune system, including monocytes, granulocytes, macrophages, and natural killer cells ${ }^{(26)}$. Therefore, prospective research that examines which type of CD11b-positive cells and which pathways are involved in BAFF production are necessary.

In conclusion, the expression of BAFF is related to increased number of $B$ cells and plasma cells, and increased production of $\lg$ A. Furthermore, BAFF-induced IgA production may be 
associated with aggravation of tissue inflammation, polyp formation and both eosinophils' aggregation and degranulation. The expression of BAFF in the subepithelial area may be associated with innate inflammatory cells (CD11b+ cells), such as monocytes, granulocytes, macrophages, and natural killer cells. Future studies are required to elucidate which cells are related in pathophysiology of this phenomenon.

\section{Authorship contribution}

YHY: JJ: KRK: SHK: KSR: YMK: not provided

\section{Conflicts of Interest}

The authors have no conflicts of interest to disclose.

\section{Acknowledgement}

This research was supported by the Basic Science Research Program through the National Research Foundation of Korea (NRF) funded by the Ministry of Education, Science and Technology (2011-0009790).

\section{References}

1. Fokkens WJ, Lund VJ, Mullol J, et al. European Position Paper on Rhinosinusitis and Nasal Polyps 2012. Rhinology Supplement. 2012(23): 1-298.

2. Van Zele T, Claeys S, Gevaert P, et al. Differentiation of chronic sinus diseases by measurement of inflammatory mediators Allergy. 2006; 61: 1280-1289.

3. Bachert C, Gevaert P, Holtappels G, Johansson SG, van Cauwenberge P. Tota and specific lgE in nasal polyps is related to local eosinophilic inflammation. J Allergy Clinical Immunol. 2001; 107: 607-614.

4. Cao PP, Li HB, Wang BF, et al. Distinct immunopathologic characteristics of various types of chronic rhinosinusitis in adult Chinese. J Allergy Clinical Immunol. 2009; 124: 478-484

5. Kato A, Peters A, Suh $L$, et al. Evidence of a role for $B$ cell-activating factor of the TNF family in the pathogenesis of chronic rhinosinusitis with nasal polyps. J Allergy Clinical Immunol. 2008; 121: 1385-1392.

6. Bachert C, Zhang N, Holtappels G, et al Presence of IL-5 protein and IgE antibodies to staphylococcal enterotoxins in nasal polyps is associated with comorbid asthma. J Allergy Clinical Immunol. 2010; 126: 962968.

7. Ng LG, Mackay CR, Mackay F. The BAFF/ APRIL system: life beyond B lymphocytes. Mol Immunol. 2005; 42: 763-772.

8. Mackay F, Silveira PA, Brink R. B cells and the BAFF/APRIL axis: fast-forward on autoimmunity and signaling. Curr Opin Immunol. 2007: 19: 327-336.

9. He B, Xu W, Santini PA, et al. Intestinal bac teria trigger $T$ cell-independent immunoglobulin $A(2)$ class switching by inducing epithelial-cell secretion of the cytokine APRIL. Immunity. 2007; 26: 812-826.

10. Xu W, He B, Chiu A, et al. Epithelial cells trigger frontline immunoglobulin class switch- ing through a pathway regulated by the inhibitor SLPI. Nat Immunol. 2007; 8: 294 303.

11. Fagarasan S, Honjo T. Intestinal IgA synthesis: regulation of front-line body defences. Nat Rev Immunol. 2003; 3: 63-72.

12. Snoeck V, Peters IR, Cox E. The IgA system: a comparison of structure and function in different species. Vet Research. 2006; 37: 455-467.

13. Kim YH, Choi BH, Cheon HG, Do MS. B cell activation factor (BAFF) is a novel adipokine that links obesity and inflammation. Ex Mol Med. 2009: 41: 208-216.

14. Rowley AH, Shulman ST, Mask CA, et al. IgA plasma cell infiltration of proximal respiratory tract, pancreas, kidney, and coronary artery in acute Kawasaki disease. J Infect Dis. 2000; 182: 1183-1191.

15. Yoshimoto K, Tanaka M, Kojima M, et al. Regulatory mechanisms for the production of BAFF and IL-6 are impaired in monocytes of patients of primary Sjogren's syndrome. Arthritis Res Ther. 2011; 13: R170.

16. Huard B, Arlettaz L, Ambrose C, et al. BAFF production by antigen-presenting cells provides $T$ cell co-stimulation. Int Immunol. 2004; 16: 467-475.

17. Kato A, Truong-Tran AQ, Scott AL, Matsumoto K, Schleimer RP. Airway epithelial cells produce $B$ cell-activating factor of TNF family by an IFN-beta-dependent mechanism. J Immunol. 2006; 177: 71647172.

18. Schneider P, MacKay F, Steiner V, et al. BAFF a novel ligand of the tumor necrosis factor family, stimulates B cell growth. J Exp Med. 1999; 189: 1747-1756.

19. Zhang N, Van Zele T, Perez-Novo C, et al. Different types of T-effector cells orchestrate mucosal inflammation in chronic sinus disease. J Allergy Clin Immunol. 2008; 122: 961-968.

20. Bossen C, Schneider P. BAFF, APRIL and their receptors: structure, function and signaling. Sem Immunol. 2006; 18: 263-275.

21. Daeron M. Fc receptor biology. Ann Rev Immunol. 1997; 15: 203-234.

22. Mihai S, Nimmerjahn F. The role of Fc receptors and complement in autoimmunity. Autoimmun Rev. 2013; 12: 657-660.

23. Woof JM, Mestecky J. Mucosal immunoglobulins. Immunol Rev. 2005; 206: 64-82.

24. Moore PA, Belvedere O, Orr A, et al. BLyS: member of the tumor necrosis factor family and B lymphocyte stimulator. Science. 1999; 285: 260-263.

25. Tribouley C, Wallroth M, Chan V, et al Characterization of a new member of the TNF family expressed on antigen presenting cells. Biol Chem. 1999; 380: 1443-1447.

26. Solovjov DA, Pluskota E, Plow EF. Distinct roles for the alpha and beta subunits in the functions of integrin alphaMbeta2. J Biol Chem. 2005; 280: 1336-1345.

Yong Min Kim, MD

Associate Professor

Department of Otorhinolaryngology-

-Head and Neck Surgery

Chungnam National University

School of Medicine

282 Munhwa-ro, Jung-Gu

Daejeon

301-721 South Korea

Tel: $+82-42-280-7696$

Fax: +82-42-253-4059

E-mail:entkym@cnu.ac.kr 